that precluding these patients from such activities is unwarranted.

The investigators analyzed data from a survey of members of the American Society of Pediatric Nephrology, and information retrieved from searches of literature databases on sports-related kidney, brain, spinal cord and cardiac injury. In total, $62 \%$ of respondents barred participation in contact/collision sports, the majority citing risk of renal injury and consequences of subsequent loss of function as primary reasons. According to the medical and sports data, cycling and skiing were the most dangerous sports in terms of damage to the kidney; American football, regarded as the most dangerous sport by $86 \%$ of the survey respondents who banned sports participation, caused only about a third the number of injuries. Many more catastrophic injuries of the spinal cord, brain, and heart were recorded than of the kidney. For all sports, the incidence of catastrophic sportsrelated kidney injury was only 0.4 per million children per year.

Grinsell et al. believe that the benefits to be gained from sports participation outweigh the small risk of renal injury, and recommend that current practices and guidelines are reviewed and clarified.

Original article Grinsell MM et al. (2006) Single kidney and sports participation: perception versus reality. Pediatrics 118: 1019-1027

\section{Sustained low-efficiency daily dialysis: safe, successful and cost-effective ARF treatment}

Sustained low-efficiency daily dialysis (SLED) has been proposed as a treatment for acute renal failure (ARF) as an alternative to intermittent hemodialysis or continuous renal replacement therapy (CRRT), high-cost modalities associated with coagulation problems, hypotension and poor fluid removal.

In an observational, prospective pilot study, the outcomes of treating 23 ARF patients with SLED were compared with those of 11 undergoing CRRT. The SLED protocol consisted of $8 \mathrm{~h}$ hemodialysis/day for 6 days/week (blood flow $200 \mathrm{ml} / \mathrm{min}$; dialysate flows $350 \mathrm{ml} / \mathrm{min}$; hemofiltration with 11 of saline per hour). Actual mean dialysis durations were $7.5 \mathrm{~h} /$ day for SLED and 21.3h/day for CRRT.
Average weekly costs were US\$1,431 for SLED with either heparin or saline flushes, $\$ 2,607$ for CRRT with heparin, and \$3,089 for CRRT with citrate. Two-thirds of SLED treatments were administered without heparin; the rate of filter clotting did not differ significantly between SLED sessions in which heparin or saline flushes were utilized.

SLED was well tolerated, and associated with markedly higher weekly $K t / V s$ than CRRT (8.4 vs $7.1 ; P<0.001)$. Time-averaged serum creatinine levels were significantly lower in the cohort of patients treated with the former modality (136 vs $95 \mu \mathrm{mol} / \mathrm{l}$ [ $1.54 \mathrm{mg} / \mathrm{dl}$ vs $1.07 \mathrm{mg} / \mathrm{dl}]$; $P=0.03)$. Ten patients treated with SLED and six treated with CRRT survived to discharge.

These preliminary results indicate that SLED is a safe, efficient and cost-effective alternative to CRRT for ARF that can be routinely performed without the need for anticoagulation.

Original article Berbece AN and Richardson RMA

(2006) Sustained low-efficiency dialysis in the ICU: cost, anticoagulation, and solute removal. Kidney Int 70: 963-968

\section{Improving access to kidney transplantation for sensitized patients: the Emory Algorithm}

Around a third of US patients awaiting renal transplantation are sensitized to HLA antigens. Sensitization greatly limits access to a transplant and, when transplant goes ahead, poses a major risk factor for subsequent antibodymediated rejection. Routine monitoring of HLA antibodies using cell-based assays-so-called panel-reactive antibody (PRA) analysis-is recommended for potential transplant recipients. The higher the percentage of targets that react with a patient's serum, the more likely there will be a positive crossmatch with a given donor and the less likely that the patient will receive a transplant. More than 15,000 patients in the US currently awaiting deceased donor kidneys are 'sensitized' (PRA $>20 \%$ ), yet only around $14 \%$ of deceased donor kidneys are transplanted into sensitized recipients.

In an attempt to find a method to help overcome this imbalance, Bray and colleagues developed a screening algorithm based on high-resolution solid-phase HLA antibody analysis to identify class $\mathrm{I} / \mathrm{II}$ antibodies, and a 'virtual crossmatch' to predict compatible or 'acceptably mismatched' donor/recipient pairs. 